\title{
FINDING THE WAY TO SUCCESS IN IMPLEMENTING LEAN CONSTRUCTION IN AN UNFAVOURABLE CONTEXT
}

\author{
Kayvan Koohestani ${ }^{1}$, Mani Poshdar ${ }^{2}$, and Vicente A. Gonzalez ${ }^{3}$
}

\begin{abstract}
Developing countries are facing sever productivity problems in their construction sectors. Lean Construction has proven to be an effective solution for such situations. However, when it comes to lean implementation, the operational attributes of the countries can cause significant impacts on the priorities, tools and techniques. This paper took Iranian construction as a sample and sought to identify and prioritise the factors that could significantly contribute to the success of the lean implementation in its context. A comprehensive literature review identified 44 critical success factors (CSFs) in a global setting. The factors were classified into four groups and were presented to industry and/ or academic professionals in the form of semi-structured open-ended interviews that enabled collecting experts' opinion about the priorities to be given. A thematic analysis of the results indicated that lack of awareness and knowledge of lean construction is the main obstacle for the Implementation. It was also established that more priority should be given to Organisational and Project Management Factors when creating Lean Construction implementation roadmap of the country. This is the first study of its type in Iran and is expected to shed light on the factors that can turn the implementation of Lean Construction a successful story, even in this unfavourable context.
\end{abstract}

\section{KEYWORDS}

Lean Construction, benefits realization, learning research, Iran, developing countries.

\section{INTRODUCTION}

Following the success of lean philosophy in manufacturing, other industries such as construction started taking advantage of its benefits to increase productivity (Shang and Sui Pheng 2014). However, due to the peculiarities of production in construction, a specific management philosophy has been tailored for construction inspired in lean (Koskela 1992). Yet, studies show that not only is construction industry peculiar and different from other sectors, but also construction industry differs from one country to another in terms of the environments in which they operate (Le-Hoai et al. 2008). When it comes to lean implementation, which means the implementation of Lean Construction

1 Head of Project Management Office at Mapna Locomotive Engineering Company, Karaj, Iran, MEng in Construction Management, The University of Auckland, koohestani@gmail.com, orcid.org/00000002-9988-3688

$2 \quad$ Lecturer, Built Envir. Engrg. Dept., Auckland Univ. of Technology, Auckland, New Zealand, mani.poshdar@aut.ac.nz, orcid.org/0000-0001-9132-2985

3 Sr. Lecturer, Department of Civil and Environmental Engineering, Founder and Research Lead Smart Digital Lab, The University of Auckland, 20 Symonds Street, Auckland, New Zealand, v.gonzalez@auckland.ac.nz, orcid.org/0000-0003-3408-3863 
practices (Sarhan et al. 2017), such differences can cause major impacts on the priorities, tools and techniques to be adopted. Therefore, while there is no a one-size-fits-all approach, it is indispensable to identify, analyse and evaluate the critical success factors of Lean Construction implementation in the unique context of each construction industry (Alkhoraif and McLaughlin 2017). This research selected the Iranian construction industry as a case study and sought to identify and prioritise the critical factors that could contribute to the success of lean implementation in its unfavourable context.

\section{THE CONSTRUCTION INDUSTRY OF IRAN}

Iran is a developing country located in the Middle East with a construction industry that plays a vital role in its economy (Ghoddousi and Hosseini 2012). After a period of decline, the output value of the country's construction sector is expected to grow at an annual average rate of $4.4 \%$ until 2023 . This growth is due the government's efforts to invest in transportation, energy and infrastructure projects (GlobalData 2019). However, despite its vast potentials, the sector has not been able to gain its deserved share of international market when compared to Turkey, a neighbouring country with comparatively similar capacities(Sabt and Mokhtariani 2016). This has been associated with the presence of several chronic problems originated by the regulatory, economic and political framework of Iran (Asnaashari et al. 2009). For instance, the country's construction sector experienced a contraction by $25 \%$ from 2013 to 2017 due to the economic recession of the country as a consequence of US sanctions (GlobalData 2019; Moghavar 2016; World Bank 2019). Besides, rules and regulations do not encourage investments within the sector, specifically from overseas (Sabt and Mokhtariani 2016). Further, systematic corruption has drastically truncated the quality of the built products (Sabt and Mokhtariani 2016). The industry also lacks adequate infrastructure and the country's political issues have restrained technology adoption. Moreover, the lack of trust amongst construction firms and unsatisfactory quality of the relationships within the industry are endemic(Sabt and Mokhtariani 2016). Such difficulties have led to a number of problems such as less foreign investment and financing, a weak private sector, shortage of resources and materials, weak infrastructure, all of which affecting the performance of the country's construction projects (Ghoddousi and Hosseini 2012; Hayati 2018; Mahdavi Adeli et al. 2013). Thereby, the Iranian construction industry has the urgency to improve productivity and competitiveness (Sabt and Mokhtariani 2016). Accordingly, Lean Construction seems to be able to provide some possible solutions.

To date, there have been some few accounts of Lean Construction exercises in Iran mostly based upon academic research. In a study by Moghavar (2016), the top three barriers of Lean Construction uptake in Iran were identified as the lack of understanding and awareness, cultural and human attitudinal issues and top management commitment. Amiri and Nazarpour (2016) reviewed the construction of school buildings in Iran and pinpointed waste identification and elimination as the most effective success factor. Also, Boroujeni and Hassani (2015) identified the Last Planner System, increased visualisation, daily huddle meetings, first-run studies, $5 \mathrm{~S}$ and mistake-proofing as six effective Lean Construction tools and techniques to increase productivity and reduce waste in Iran.

Accordingly, it seems that the implementation of Lean Construction in the unfavourable context of the Iranian construction has its specific challenges that need to be exclusively studied and addressed. 


\section{RESEARCH METHOD}

This research conducted an extensive round of literature review to identify the CSFs of Lean Construction implementation in a global context. The review used Google Scholar as its primary database with the search strings included by "lean construction" AND "success factor" OR "enabler" OR "driver". In order to involve the most recent studies published in the past five years, the publication time was set to be after 2014. This round returned a total number of 156 journals, conference papers, theses and dissertations. Afterwards, a content analysis through abstract reading refined the results to those related to the critical factors of success in the implementation of Lean Construction. All other studies that had covered the lean topics in different contexts such as manufacturing or those with no relation to Lean Construction were excluded. The refined results, which amounted to 41 papers, were reviewed and coded for analysing CSFs by using NVivo software package (QSR International) which also enabled us to keep track of the frequency of reports on each factor. The list of the identified factors was then evaluated to ensure its comprehensiveness as well as the uniqueness and proper naming of the identified factors. Afterwards, a set of semi-structured open-ended interviews was undertaken to obtain the perception of the field experts about the priority and importance of the identified CSFs in the context of the Iranian construction industry. This type of interviews enabled spontaneous communications to investigate the operational knowledge of the experts gained from years of practice (Bogner et al. 2009). Accordingly, five face-to-face interviews were conducted. The responses were transcribed and developed into coherent essays suitable for thematic analysis in NVivo. The results provide an insight into the perception of the experts about the priorities to be given to the CSFs when creating the road map of Lean Construction implementation of the country.

\section{SAMPLING DETAILS}

Qualified respondents were determined to have previous exposures to lean thinking and lean philosophy (either through practical implementation, academic courses or training). They were also needed to have a work experience of at least five years in the Iranian construction industry. An online search was conducted to identify qualified respondents. Due to the scarcity of the professionals with the knowledge of lean and familiarity with the local construction industry, the criteria returned an extremely restricted number of cases. Within the three months of study, ten interview requests were sent out using emails, phone calls and instant messaging. Five were accepted that equates to a response rate of $50 \%$. The restricted size of the study sample made a quantitative analysis of the results impractical. Accordingly, the responses were analysed only qualitatively. Table 1 presents the respondents' demographic information.

\section{INTERVIEWS}

A questionnaire along with a list of the identified factors was prepared. The questionnaire included the following three questions:

- To what extent have the factors on the list been applied in the Iranian construction industry?

- Which of the factors could play a significant role in the success of lean construction implementation in the country?

- What would other factors and/ or lean tools be suggested by the interviewees to include? 
The interview sessions started with a short briefing on the research method and explaining the intended meaning of each factor included in the presented list. These two conducts ensured that no misunderstanding would happen about the factors in the process of interview. The average time of the five interviews was calculated to be 68 with the minimum of 46 and maximum of 88 minutes.

Table 1: Demographic Information of the Respondents

\begin{tabular}{ccccccc}
\hline \# & Job Title & $\begin{array}{c}\text { Years in } \\
\text { Industry }\end{array}$ & $\begin{array}{c}\text { Years in } \\
\text { Academia }\end{array}$ & Education & Major & Province \\
\hline 1 & $\begin{array}{c}\text { Assistant } \\
\text { Professor }\end{array}$ & 11 & 7 & PhD & $\begin{array}{r}\text { Construction } \\
\text { Management }\end{array}$ & Tehran \\
& $\begin{array}{c}\text { Project } \\
\text { Manager }\end{array}$ & 9 & 0 & Master's & $\begin{array}{r}\text { Construction } \\
\text { Management }\end{array}$ & Tehran \\
3 & Tutor & 15 & 4 & PhD & $\begin{array}{r}\text { Construction } \\
\text { Management }\end{array}$ & Tehran \\
& $\begin{array}{c}\text { Project } \\
\text { Planner }\end{array}$ & 13 & 0 & Master's & $\begin{array}{r}\text { Construction } \\
\text { Management }\end{array}$ & Alborz \\
& Lecturer & 7 & 1 & PhD & $\begin{array}{r}\text { Industrial } \\
\text { Engineering }\end{array}$ & Fars \\
\hline
\end{tabular}

\section{CRITICAL SUCCESS FACTORS OF LEAN CONSTRUCTION}

The literature review indicated a total number of 44 Lean Construction implementation CSFs categorized into 4 groups of Organisational Factors, Project Management Factors, External Factors and factors related to Implementation of Lean Practices.

Organisational Factors consist of those that are decided, determined or formed at the business level. The total number of references addressing this category was 35 . They include: 1) Management and Leadership: commitment and support, decision making, facilitation and leadership, Freq: 26, e.g. (Demirkesen and Bayhan 2019); 2) Human Resource: involvement, motivation, skills and knowledge, Freq: 25, e.g. (Zhang et al. 2017); 3) Training: increasing lean awareness and knowledge through training, Freq: 17, e.g. (Daniel et al. 2019); 4) Technology Adoption and Infrastructure: investment on of modern infrastructure to support lean practices, Freq: 16, e.g. (Aljobaly and Banawi 2019); 5) Organizational Culture and Teamwork: supportive culture to promote coordinated teamwork, Freq: 15, e.g. (Daniel et al. 2019); 6) Organizational Inertia and change management: resistance to change and the ability to manage change, Freq: 14, e.g. (Sarhan 2018); 7) Organizational Strategy and Policies: whether lean policies are linked to long-term goals, Freq: 11, e.g. (Demirkesen and Bayhan 2019); 8) Funding: the amount invested in lean practices, Freq: 8, e.g. (Aljobaly and Banawi 2019); 9) Organizational Structure: structures that support communication and teamwork and reduce bureaucracy, Freq: 4, e.g. (Omotayo et al. 2018); 10) Firm's Marketing and Market Share: clear marketing vision and the firm's share of market, Freq: 2, e.g. (Demirkesen and Bayhan 2019); 11) Firm's Financial Status: company's turnover that affects its ability to support and fund lean implementation, Freq: 2, e.g. (Omotayo et al. 2018); 12) Competitive Advantage: the power to win new contracts, Freq: 1, e.g. (Noor et al. 2018). 
Project Management Factors consist of those which have implications for Project Management and are decided, determined or formed at project level. The total number of references addressing this category was 37. They include: 13) Communication, Information Exchange, Learning and Knowledge Management: increased transparency through effective communication and information exchange, learning from mistakes and use of knowledge management systems, Freq: 22, e.g. (Saini et al. 2018); 14) Supply Chain Management (SCM)- Relationships: Improved relationships to enhance cooperation, avoid conflicts and establish trust, Freq: 18, e.g. (Omotayo et al. 2018); 15) Stakeholders Management: early stakeholders involvement, stakeholder's awareness of LC, Freq: 17, e.g. (Daniel et al. 2019); 16) SCM- Integration: reduced fragmentation by Improving coordination and collaboration, Freq: 15, e.g. (Zhang et al. 2017); 17) Scope and Requirements Management: clear scope definition, value identification and definition from customers' point of view, Freq: 10, e.g. (Sarhan 2018); 18) SCM-Procurement and Contracting Strategy: support of lean practices by tendering mechanisms and type of procurement, Freq: 9, e.g. (Zhang et al. 2017); 19) Risk and HSE Management: Includes project risk as well as safety and health management, Freq: 9, e.g. (Aljobaly and Banawi 2019); 20) Resource Management: planning, estimation, acquisition, allocation and control of resources, Freq: 9, e.g. (Zhang et al. 2017); 21) Planning and Scheduling: inadvance work planning and realistic scheduling using lean techniques, Freq: 8, e.g. (Aljobaly and Banawi 2019); 22) SCM-Commitment to Promises: firm's commitment to contractual obligations and promises, Freq: 6, e.g. (Daniel et al. 2019); 23) Cost Management: cost planning, estimation, budgeting, monitoring and control, Freq: 3, e.g. (El-Abidi et al. 2019); 24) Quality Management: quality planning, performing and control, Freq: 3, e.g. (Zhang et al. 2017); 25) SCM-Relationship Management: claims handling, dispute resolution, Freq: 2, e.g. (Omotayo et al. 2018); 26) SCM-Material Handling, Logistics and Transportation: on-time material delivery, optimised material transportation, Freq: 2, e.g. (El-Abidi et al. 2019); 27) Project Manager Involvement: The degree to which project manager is involved lean implementation, Freq: 1, e.g. (Tayeh et al. 2018).

External Factors consist of those that are formed, determined or decided as a consequence of the environment in which the firm or the project operates. The total number of factors addressing this category was 17 . They include: 28) Government and Regulatory Bodies: policies and regulations to encourage the use of lean practices, Freq: 9, e.g. (Sarhan 2018); 29) Local Construction Industry: relationships between players, market competition, relationships with academia, corruption Freq: 9, e.g. (Aljobaly and Banawi 2019); 30) National Economy: economic stability, inflation, support from national economy Freq: 4, e.g. (Amuda-Yusuf 2018); 31) Resource Availability: availability of resources to implement project activities and support lean practices Freq: 4, e.g. (Zhang et al. 2017); 32) External Stakeholders Influence: client push to adopt and implement lean practices, influence of stakeholders' behaviour Freq: 4, e.g. (Demirkesen and Bayhan 2019); 33) Weather and Environment: the impact of environmental factors on workflow reliability Freq: 3, e.g. (Zhang et al. 2017); 34) Applicability of Lean Tools and Techniques: simplicity and applicability of lean tools and techniques Freq: 2, e.g. (Aljobaly and Banawi 2019); 35) Project Type and Complexity: influence of project complexity on the implementation of lean practices Freq: 2, e.g. (Demirkesen and Bayhan 2019).

Factors related to the Implementation of Lean Practices consist of haphazard implementations of Lean Construction practices without prior conception of its principles. 
The total number of factors addressing this category was 25. They include: 36) Process Improvement and Standardization, Freq: 16, e.g. (Omotayo et al. 2018); 37) Lean Design Management: design integration, change minimization and design standardization, Freq: 11, e.g. (Aljobaly and Banawi 2019); 38) Benchmarking and Performance Measurement, Freq: 7, e.g. (Daniel et al. 2019); 39) Continuous Improvement, Freq: 6, e.g. (Omotayo et al. 2018); 40) Prefabrication, Modularization and Standardization, Freq: 4, e.g. (El-Abidi et al. 2019); 41) $R \& D$ and Innovation: more efficient processes, materials, etc. as a result of research and development activities, Freq: 4, e.g. (El-Abidi et al. 2019); 42) Early Lean Involvement: involvement of Lean Construction from design stage, Freq: 2, e.g. (Sarhan 2018); 43) Suitable Lean Implementation Strategy: use of an evolutionary approach with clear goals for each step, Freq: 2, e.g. (Amuda-Yusuf 2018); 44) Assessment of lean Barriers: identification and assessment of lean barriers and development of an action plan for each, Freq: 1, e.g. (S.A.Ward 2015).

The most frequent categories in the literature review consisted of Organisational and Project Management Factors. If the frequency factor is regarded as the priority in the global context, Organisational and Project Management Factors could be perceived as the most prior global categories. Also, with the same hypothesis, Management and Leadership, Human Resource, Communication and Knowledge Management, Supply Chain Relationships, Training and Stakeholders Management could be seen as the top most frequent global factors.

\section{RESULTS AND DISCUSSIONS}

\section{Current Status of Organizational Factors}

A thematic analysis of the responses in NVivo identified the followings:

Management and Leadership: The Iranian construction companies, especially in the private sector, are known for being highly influenced by the charisma of their top managers. Thus, the degree of involvement in lean adoption and implementation would purely depend upon the decision of the managers. Yet, responses indicated a low level of awareness about the advantages of lean implementation, especially amongst the managers who typically perceive waste as an inherent aspect of construction projects. Hence, the factors related to management and leadership were recognized by the interviewees to be the most effective amongst others.

Human resource: The role of human resource was perceived as almost neglected in the industry. The interviewees identified construction firms to regard employees as abundant inexpensive resources. Thus the employers tend to keep the wages low that limits the devotedness of the employees in turn.

Organisational Inertia and Change Management: As long as awareness about the benefits of innovative approaches remains low, a high resistance to change could be expected. Therefore, organizational inertia was detected to be a significant problem. Effective change management was also perceived to be a rare practice in the Iranian organisations.

Training: Due to the low level of awareness about the advantages of Lean Construction, the respondents emphasized the importance of training. Nevertheless, they had perceived construction companies as not allocating enough budget to train their personnel. The quality of the training and assessments were also reported to be inadequate.

Technology Adoption and Infrastructure: Despite acknowledging the Iranian construction companies to be open to adopting technology, the process was realized to be 
traditional and far from the lean approach. This is because the companies were recorded to confuse technology adoption with automation as they would typically neglect to plan and strengthen their processes before adopting new technologies. On the other hand, the motivation behind technology adoption, in some cases, might only be to stay fashionable and attract clients. It was believed that technology adoption results in no significant improvement in this situation.

Organisational Culture and Teamwork: While the culture of working in teams found to be weak amongst Iranian construction firms, it was believed to be jeopardised by the existence of multiple unnecessary organisational interfaces and the quality of relationships between Iranian construction firms.

Organizational Strategy and policies: According to the respondents, very few of the Iranian construction firms would involve strategic planning and have their mission and vision statements documented. This situation might have arisen from the country's political and economic situation, which makes the long-term planning a challenging task. Also, the interviewees discerned the setup of the organizational policies not supportive of lean implementation. The interviewees addressed the bureaucratic structure of the work procedures in the public sector as well as the decision making structure in the private sector as typical circumstances that expose the waste-producing nature of the policies.

Funding: At the time of the study, several Iranian construction companies were dealing with major financial problems, and this had made the investment in improvements a rare practice. Nevertheless, among the firms with better financial status, no tendency was found to invest in lean implementation. The interviewees related this situation to the lack of proper understanding and knowledge of Lean Construction amongst the managers.

Organizational Structure: The interviewees notified that most of the construction companies of the country lack a documented organizational structure. Accordingly, no clear definition would be found about the roles and responsibilities. Also as mentioned earlier, teamwork is hindered by the existence of multiple unnecessary interfaces.

Marketing, Market Share and Competitive Advantage: The companies recognize the power of lobbying as the competitive advantage to win new contracts. In the meantime, it was recorded that the firms with lower market share, especially the younger ones, still need to increase their competitive advantage by embracing modern and innovative approaches. Nevertheless, this is hindered by the lack of awareness of lean philosophy and its practices amongst these companies.

\section{Current Status of Project Management Factors}

Communication, Information Exchange, Learning and Knowledge Management: The construction companies of the country found highly restrained in sharing their information. Communications were reported to be limited to the contractual obligations, mostly through basic modes such as face to face meetings, sending letters or periodic reports. The introduction of instant messaging services has not resulted in major improvements in communications between project parties.

Learning and Knowledge Management: A small number of companies were realised to have Knowledge management in place through the use of Project Management Information Systems (PMIS). However, the use of such systems has not typically been supported by the project managers which means that although a very limited number of firms have the infrastructure in place, the systems are barely used by project team members to record the project knowledge and lessons learned. This was again linked with 
the lack of awareness amongst project managers. As a result, knowledge is not preserved and is lost when people leave the organisations.

Supply Chain Management: While this was reported to be a significant challenge, prevailing practices in this area were known to be traditional. Procurement was understood to be still centred on outdated methods. The dominant local monopolies would control procurement by manipulating the supply market. In this situation, the adoption of lean approaches such as Just in Time (JIT) was found to be barely possible and instead, the firms tend to fill up their inventory upon finding the prices stable. Also, stock keeping was noted to be limited to large-scale projects. However, as mentioned, even in these projects, lean inventory management and material flow practices such as Kanban and JIT were rare. The material flow was found to be discrete and its planning was limited to particular cases such as transporting large steel structural elements.

The public sector owns the majority of the country's projects, in which they would contract the projects to the private sector. At the time of the study, no contracting strategy was observed among the firms, where rigid traditional contractual terms defined relationships between the parties. Accordingly, the firms would thrive on taking full advantage of the contractual commitments. In this environment, claims were prevalent, relationships were adversarial and there was no account of relational contracting. Most contractors were reported to use only the price and duration as their tender evaluation measures, especially in the public sector. However, relationships were found to be slightly better in projects owned by the private industry. In large-scale projects, jobs were realised sometimes to be implemented without the contracts being signed before. These practices were found to be typical when the contractor and sub-contractor had previous work relationships, or when the contractor was highly credited within the industry. Contracting was reported to be even less common in small-scale private projects, where works were mostly performed only based upon oral agreements.

Stakeholder management: This was found as a rare practice as it is mostly cantered on corrupt activities to minimize the undesired influence of supervisory bodies which inspect the compliance of the construction works with regulations.

Quality Management: The country's construction firms were realised to have little understanding of the basic quality concepts. The practices were mostly limited to the use of checklists to inspect quality failures. No plan or procedure was found to be in place in particular to quality assurance and quality management.

Scope and Requirements Management: A lack of clear scope and requirements definition were also reported. The interviewees related the misevaluations of deliverables with these ambiguities which were also considered responsible for the frequent change orders that produce substantial waste and rework.

Risk and HSE Management: Implementation of risk management was reported to be seen in large-scale projects. However, as the identification, evaluation, analysis and risk treatment would not follow a structured system, no significant improvements had been reported from this implementation. As a standard practice, the large-scale projects would involve a Health, Safety and Environment (HSE) department to comply with regulations. Nevertheless, the departments were not empowered to control operations and little planning was conducted for the health and safety management of projects.

Resource Management: Despite the practice of resource planning in multi-project organizations, resource management and resource optimization were considered as scarce. Schedules were barely checked against resource availability. Therefore, work interruption due to resource conflicts was found to be common in projects. 
Planning and Scheduling: This was found to be a common practice in large-scale projects. However, even in these cases, project schedules are mostly used for tracking progress rather than forward planning. Therefore, the plans remain unrealistic and unreliable.

Cost Management: The majority of the construction companies of the country were perceived to misinterpret the project cost management. They were found to usually confuse it with organizational cost/ financial management and thus, their cost management strategy was unable to support the project level.

Project Manager's Involvement: Project managers were understood to be unaware of the lean philosophy, which significantly prevents them to be involved in and undertake any lean initiatives in their projects.

\section{Current Status of External Factors}

All respondents agreed that external factors could influence the lean implementation.

Government and Regulatory Bodies: International sanctions were considered as a source of shortage in equipment and material supply. Firms were known to keep substantial inventories with the hope of mitigating the risk of supply shortage and preventing unwanted delays. In addition, international sanctions would impact resource availability as it would prevent the import of construction machinery and equipment to undertake modern construction methods. This, in some cases, would lead to delays, cost overruns or quality reductions due to the usage of labour-, time- and cost-intensive methods in place of modern approaches.

National Economy: The economic downturn of the country, has led to various financial problems for construction firms. As discussed, such difficulties reduce the firm's tendency to invest in improvement initiatives such as Lean Construction practices.

Local Construction Industry: As discussed, in many cases local monopolists control the supply market. They were seen to be responsible for manipulating the market price of strategic materials such as steel and cement. As a result, prices would fluctuate and the market might experience shortage of materials at certain periods. Competitions were found to be the slightest and limited to price races. The interviewees perceived it to undermine the lean implementation. Typically, low prices would lead to low quality and delayed deliveries. There is also the role of Iran Construction Engineering Organization which supervises and inspects all building construction projects in the country. The conflicting roles of this entity along with its monopolistic situation result in conflicting interests which leads to vast systematic corruption within the industry.

Project Type, Weather and Environment: The interviewees considered these as low-influence factors. In the case of weather and environment, it was argued that they can be influential for areas that suffer from harsh climate during hot and cold seasons.

\section{Current Status of Factors of Haphazard Implementation of Lean Practices}

The responses indicated that the industry had implemented some of the available lean approaches. The implementation, however, was considered to be highly intuitive and to a limited extent. For instance, benchmarking was found where the companies would check the new methods adopted by their competitors. Moreover, prefabrication was realised to be known for several years despite being adopted in a non-lean way. An interviewee referred to a personal experience during which huge piles of components were prefabricated and stored for long durations on-site waiting to be assembled. The responses also showed that the level of innovation was extremely low within the construction 
industry of the country. One interviewee also referred to BIM as an available practice at some construction sites.

\section{OTHER FACTORS}

No other factor was proposed by the respondents. This indicates that the interviewees agree with the comprehensiveness of the identified factors.

\section{MAJOR FACTORS FOR THE SUCCESS OF LEAN IMPLEMENTATION IN THE IRANIAN CONSTRUCTION INDUSTRY}

The interviewees identified management and leadership, human resources and training as the most significant CSFs of the lean implementation at the organizational level. This was perceived to be due to the root cause of little knowledge of lean philosophy and awareness of the benefits of implementing its practices. Scope management, relationships between parties and communications were indicated as the most crucial CSFs in the lean implementation at project level. The findings indicate the crucial role of top managers and project managers in the successful implementation of lean construction practices. Also the majority of participants referred to the significant role that the governments and regulatory bodies could play in promoting lean implementation by providing incentives, supporting awareness and maintaining stability.

These findings are highly consistent with the priority of the factors according to their frequency in the studied literature.

\section{SUMMARY AND RECOMMENDATIONS}

A study was conducted to identify the important CSFs in implementing Lean Construction in the Iranian construction industry. It established a basic framework for lean implementation in a developing country with highlighting the significant role of governments, top managers and project managers. The results suggested that organizational and project-related factors have a higher influence on the success of the implementation of Lean Construction than External Factors and the factors related to haphazard implementation of lean tools and techniques. Six factors were identified that must be closely monitored to achieve a successful Lean Construction implementation in Iran. A review of these factors indicates that increasing knowledge and awareness about the benefits of Lean Construction can present a crucial start point, which is in line with other studies of lean in the public sector. Governments and regulatory bodies can help to speed up the process. It was also found that in order to maintain a proper level of competitiveness, young companies can undertake innovative approaches. Therefore, they can potentially act as the pioneers of adopting Lean Construction in the industry. Given the significance of financial aspects for such companies, emphasizing on the cost-saving capability of Lean Construction can serve as an important incentive to promote its implementation.

Research limitations: the literature review was conducted on the basis of publications dated after 2014 and it did not include papers which studied lean CSFs in other industries. Online information was the sole reference to identify potential candidates and sampling criteria was narrowed down to those with prior exposure to Lean Construction and a minimum of five years of work experience in the Iranian construction industry.

Future research could consider a quantitative investigation of the identified critical success factors. The study could also expand on the background of those project managers with either no prior information of Lean Construction or those who are informed of the 
Lean Construction concept, but are not in favour of its implementation. Furthermore, the most effective Lean Construction tools and techniques to address the CSFs and barriers could be identified and analysed in future studies. Also another area to consider for further research would be to discover the interrelationships and dependencies between the CSFs of Lean Construction implementation. Lastly, the role of Lean Construction to discourage corruption in such contexts could be included in future research.

\section{ACKNOWLEDGMENT}

The authors would like to thank all the academics and experts who took time and shared their valuable views during interviews for the purpose of this study.

\section{REFERENCES}

Aljobaly, O., and Banawi, A. (2020). "Evaluation of the Saudi Construction Industry for Adoption of Building Information Modelling." Proc. International Conference on Applied Human Factors and Ergonomics, Springer, 488-498.

Alkhoraif, A., and McLaughlin, P. (2017). "Organisational culture-Enablers and inhibitors factors for the effective implementation of lean." International Journal of Lean Thinking, 8(2), 65-96.

Amiri, M. J. T., and Nazarpour, H. (2016). "Check the Status of Lean Construction in Iran Civil Projects, Case Study: School Renovation Office of Golestan province Projects." Journal of Golestan Construction, 5(2), 75-85.

Amuda-Yusuf, G. (2018). "Critical success factors for building information modelling implementation." Construction Economics and Building, 18(3), 55-73.

Asnaashari, E., Knight, A., Hurst, A., and Farahani, S. S. (2009). "Causes of construction delays in Iran: project management, logistics, technology and environment." Proc. 25th Annual ARCOM Conference, Nottingham, UK, 897-906.

Boroujeni, K. A., and Hassani, H. (2015). "Lean Construction: From Theory to Implementation." International Journal of Academic Research, 37-49.

Daniel, E. I., Pasquire, C., and Dickens, G. (2019). "Development of Approach to Support Construction Stakeholders in Implementation of the Last Planner System." Journal of Management in Engineering, 35(5), 04019018.

Demirkesen, S., and Bayhan, H. G. (2019). "Critical Success Factors of Lean Implementation in the Construction Industry." IEEE Transactions on Engineering Management, 1-17.

El-Abidi, K. M. A., Ofori, G., Zakaria, S. A. S., Mannan, M. A., and Abas, N. F. (2019). "Identifying and Evaluating Critical Success Factors for Industrialized Building Systems Implementation: Malaysia Study." Arabian Journal for Science and Engineering, 44(10), 8761-8777.

Ghoddousi, P., and Hosseini, M. R. (2012). "A Survey of the Factors Affecting the Productivity of Construction Projects in Iran." Technological and Economic Development of Economy, 18(1), 99-116.

GlobalData (2019). "Construction in Iran - Key Trends and Opportunities to 2023." GlobalData, Londo, United Kingdom, 48.

Hayati, F. K. N. A., Majid; Andersen, Maria Reinholdt; Atamanov, Aziz; Wai-Poi, Matthew Grant; Mostafavi Dehzooei, Mohammadhadi; Salehi Isfahani, Djavad; (2018). "Iran Economic Monitor: Weathering Economic Challenges (English)." World Bank Group, Washington, D.C, USA. 
Koskela, L. (1992). Application of the New Production Philosophy to Construction. Stanford University, Stanford, California.

Le-Hoai, L., Dai Lee, Y., and Lee, J. Y. (2008). "Delay and cost overruns in Vietnam large construction projects: A comparison with other selected countries." KSCE Journal of Civil engineering, 12(6), 367-377.

Mahdavi Adeli, M., Eskandari Malayeri, M., Naghavi, O., and Akbari, R. (2013). "New methods for captive finance in project based companies using private sectors supply." European Online Journal of Natural and Social Sciences, 2(3), 863-869.

Moghavar, E. M. (2016). "Identifying the Barriers of Implementing Lean Construction Principals in Developing Countries." Master's Thesis, Middle East Technical University, Turkey.

Noor, S. R. M., Yunus, R., Abdullah, A. H., Nagapan, S., Syahir, S. M., and Mazlan, S. (2018). "Insights into The Adoption of Lean Management in Industrial-ised Building System (IBS) Implementation: The Drivers and Challenges." International Journal of Engineering \& Technology, 7(3.23), 22-31.

Omotayo, T. S., Kulatunga, U., and Bjeirmi, B. (2018). "Critical success factors for Kaizen implementation in the Nigerian construction industry." International Journal of Productivity and Performance Management, 67(9), 1816-1836.

QSR International. 2018. NVivo Qualitative Data Analysis Software, version 12, Australia.

Sabt, M.H., and Mokhtariani, M. (2016). "An Evaluation and Analysis of Iran's Construction Industry and Presenting a Number of Solutions for Improvement." Amirkabir Journal of Science and Research, 4(6), 46-59.

Saini, M., Arif, M., and Kulonda, D. J. (2018). "Critical factors for transferring and sharing tacit knowledge within lean and agile construction processes." Construction Innovation, 18(1), 64-89.

Sarhan, J.G.I. (2018). "Development of a lean construction framework for the Saudi Arabian construction industry." PhD Diss., Queensland University of Technology, Australia.

Sarhan, J.G., Xia, B., Fawzia, S., and Karim, A. (2017). "Lean construction implementation in the Saudi Arabian construction industry." Construction Economics and Building, 17(1), 46-69.

Shang, G. and Sui Pheng, L. (2014). "Barriers to lean implementation in the construction industry in China." Journal of Technology Management in China, 9(2), 155-173.

Tayeh, B.A., Al Hallaq, K., Al Faqawi, A.H., Alaloul, W.S., and Kim, S.Y. (2018). "Success Factors and Barriers of Last Planner System Implementation in the Gaza Strip Construction Industry." The Open Construction and Building Technology Journal, 12(1).

Ward, S.A. (2015). “Critical Success Factors for Lean Construction Intervention.” PhD Diss., The University of Dundee, Dundee, Scotland.

World Bank (2019). "Iran's Economic Update - April 2019." World Bank Group, $<$ https://www.worldbank.org/en/country/iran/publication/economic-update-october2019>.

Zhang, L., Chen, X., and Suo, Y. (2017). "Interrelationships among critical factors of work flow reliability in lean construction." Journal of Civil Engineering and Management, 23(5), 621-632. 